\title{
Clinical epidemiology of gastric cancer
}

Tiing Leong $\underline{A n g}^{1}$, MBBS, FRCP, Kwong Ming $\underline{\text { Fock }}^{1}$, MBBS, FRCP

\begin{abstract}
Gastric cancer is the second leading cause of cancer-related mortality and the fourth most common cancer globally. There are, however, distinct differences in incidence rates in different geographic regions. While the incidence rate of gastric cancer has been falling, that of gastric cardia cancers is reportedly on the rise in some regions. Helicobacter pylori (H. pylori) infection is a major risk factor of non-cardia gastric cancer, and data has emerged concerning the role of $\mathrm{H}$. pylori eradication for primary prevention of gastric cancer. Dietary, lifestyle and metabolic factors have also been implicated. Although addressing these other factors may contribute to health, the actual impact in terms of cancer prevention is unclear. Once irreversible histological changes have occurred, endoscopic surveillance would be necessary. A molecular classification system offers hope for molecularly tailored, personalised therapies for gastric cancer, which may improve the prognosis for patients.
\end{abstract}

Keywords: epidemiology, gastric cancer, H. pylori

\section{INTRODUCTION}

Gastric cancer is the second leading cause of cancer-related mortality and the fourth most common cancer globally. However, its incidence rates in different geographical regions are distinctly varied. Aetiologically, gastric cancer is associated with Helicobacter pylori (H. pylori) infection, dietary and lifestyle factors, and genetics. This review provides an update of the clinical epidemiology of gastric cancer.

\section{EPIDEMIOLOGICAL TRENDS IN GASTRIC CANCER}

The latest estimate of the global incidence and mortality rates of gastric cancer was updated by the International Agency for Research on Cancer (IARC) in 2008, through its GLOBOCAN series (Fig. 1). ${ }^{(1,2)}$ This updated report estimated that there were 989,000 new cases of gastric cancer (or $7.8 \%$ of all reported cancer cases) in 2008. This estimate made gastric cancer the fourth most common malignancy globally, behind lung, breast and colorectal cancers. Significantly more gastric cancer cases were noted in less developed regions compared to more developed regions. In the male gender, 466,900 cases were reported in less developed regions compared to 173,700 cases in more developed regions. The corresponding case burden for the female gender was 247,000 and 102,000 cases, respectively.

About half of the total gastric cancer load was located in East Asia, especially China. The age-standardised incidence rate (ASIR) for males was generally twice that for females. The cancer risk in different geographical regions has been defined based on the magnitude of the ASIR. ${ }^{(3)}$ A high-risk region is defined as ASIR > 20 per 100,000, an intermediate-risk region as 10-20 per 100,000, and a low-risk region as < 10 per 100,000. The ASIR of the different geographical regions, subdivided by gender, can be accessed at the GLOBOCAN website. ${ }^{(2)} \mathrm{A}$ key point to note is that, in general, most of the high-risk regions are located in East

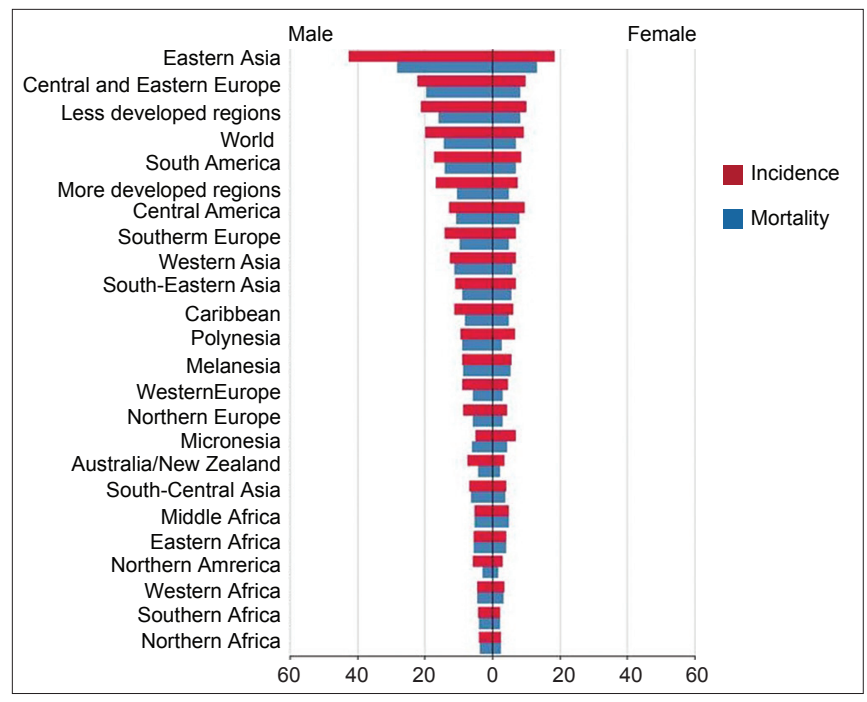

Fig. 1 Graph shows the estimated gastric cancer age-standardised incidence and mortality rates per 100,000 (reproduced from http://globocan.iarc. $\left.\mathrm{fr} /{ }^{(2)}\right)$

Asia. In particular, the ASIR among males in Korea, Mongolia, Japan and China is especially high, with the ASR at 62.2, 48.2, 46.8 and 41.3 per 100,000 , respectively. ${ }^{(2)}$

Geographic variations in gastric cancer incidence also exist within a country. For instance, in China, the risk of gastric cancer in Changle, Fujian, was fivefold that of Hong Kong.(4) Muping County in Shandong, China also had a significantly higher risk of gastric cancer when compared to Yanqing County in Beijing. ${ }^{(5)}$ Geographic variations in gastric cancer incidence and mortality in terms of a north-south gradient have been reported in both the West and the East. ${ }^{(6-8)}$ These variations may be related to differences in risk factors, such as the prevalence rate of $H$. pylori infection. Migration studies have shown that first-generation migrants from countries with a high incidence of $H$. pylori infection relocating to countries of low incidence rates had similar risks as that of the country of origin, but the incidence rate

${ }^{1}$ Department of Gastroenterology and Hepatology, Changi General Hospital, Singapore

Correspondence: A/Prof Ang Tiing Leong, Senior Consultant and Chief, Department of Gastroenterology and Hepatology, Changi General Hospital, 2 Simei Street 3, Singapore 529889. tiing_leong_ang@cgh.com.sg 
tended toward that of the host country in subsequent generations, suggesting the important role of environmental risk factors. ${ }^{(9-12)}$

Up till the mid-1990s, gastric cancer was the most common cause of cancer-related mortality globally. Gastric cancer is currently the second leading cause of cancer-related mortality for both genders globally, constituting $9.7 \%$ of all cancer-related deaths. The highest age-standardised mortality rate (ASMR) is estimated in Eastern Asia (28.1 per 100,000 in men, 13.0 per 100,000 in women), the lowest ASMR in North America (2.8 and 1.5 per 100,000 , respectively). High mortality rates are also noted in both genders in Central and Eastern Europe, and in Central and South America. ${ }^{(2)}$ The higher mortality rates in these geographical regions are a reflection of a greater disease burden due to higher incidence rates. It is noteworthy that, although Korean and Japanese males were ranked as having the highest and third highest incidence rates for gastric cancer globally, they were ranked 12th and 16th, respectively, in terms of mortality rates. A similar trend can be seen for females in these two countries. ${ }^{(2)}$

The incidence (Fig. 2) and mortality rates (Fig. 3) of gastric cancer have continued to fall over the course of time. This decreasing trend has been observed in both developed and developing regions, regardless of their risk for gastric cancer. ${ }^{(13-17)}$ The United States of America (USA) is a developed country with an overall low incidence rate of gastric cancer, although actual risk may vary due to ethnicity. The gastric cancer data for the USA from 1977 to 2006 was recently analysed - the overall ASIR per 100,000 population was found to have declined during the study period, from 5.9 (95\% confidence interval [CI] 5.7-6.1) to $4.0(95 \% \mathrm{Cl} 3.9-4.1)$ in whites, and from 13.7 (95\% Cl 12.5-14.9) to 9.5 (95\% Cl 9.1-10.0) in blacks. ${ }^{(16)}$ The trends in gastric cancer mortality data from Japan (a country with high gastric cancer risk), and Hong Kong and Singapore (countries with intermediate gastric cancer risks) revealed a continued decrease in ASMR from 1950 to 2004. ${ }^{(15)}$ Bertuccio et al, ${ }^{(13)}$ who analysed global gastric cancer mortality from 1980 to 2005 using joinpoint regression analysis, provided updated site-specific incidence rates from 51 selected registries. It was found that the annual percent change (APC) in mortality rate was around $-3 \%$ to $4 \%$ for the major European countries, which was similar to countries such as Korea $(-4.3 \%)$, Australia $(-3.7 \%)$, the USA $(-3.6 \%)$, Japan $(-3.5 \%)$, Ukraine $(-3 \%)$ and the Russian Federation (-2.8\%). In Latin America, the APC was slightly lower: Brazil and Chile (-1.6\%); Argentina and Mexico $(-2.3 \%)$; Colombia $(-2.6 \%) .{ }^{(13)}$ Bray et al ${ }^{(17)}$ used the Human Development Index (HDI), a composite indicator of life expectancy, education and gross domestic product per head, to highlight cancer-specific patterns in 2008 (on the basis of GLOBOCAN estimates) and trends in 1988-2002, and to produce future burden scenario for 2030. It was estimated that there will be a $2.3 \%$ annual decrease in gastric cancer incidence rates till 2030. ${ }^{(17)}$

The temporal decrease in incidence rates of gastric cancer has been ascribed to reduction in $H$. pylori infection in successive birth cohorts, which is related to changing childhood environment, improved food preservation practices such as the use of refrigeration as opposed to salt, and better nutrition. Gastric

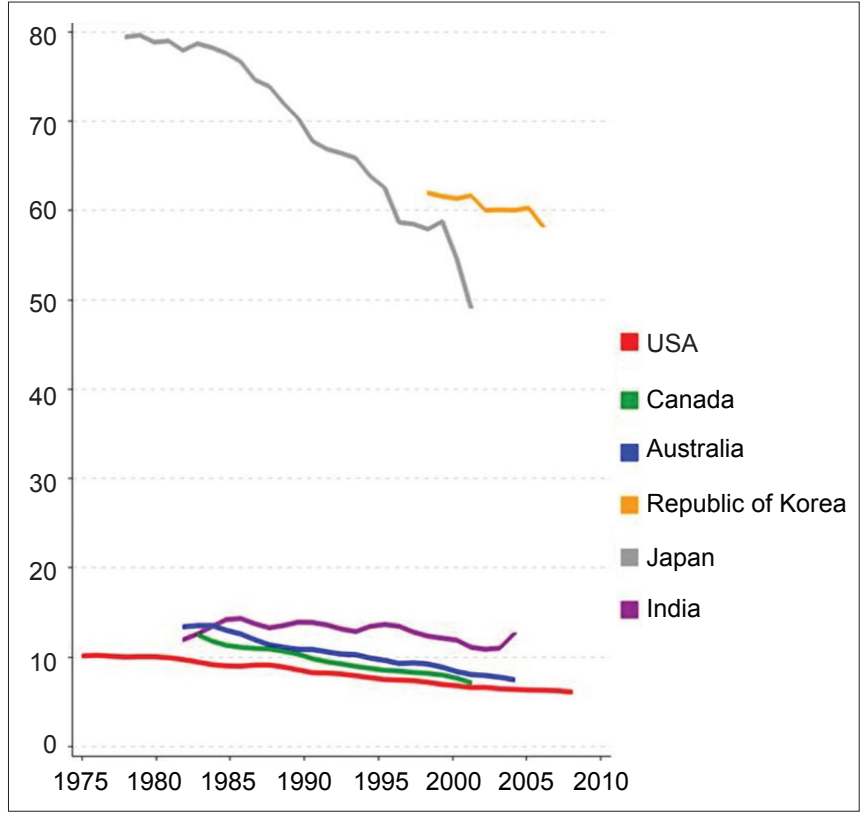

Fig. 2 Graph shows the trends in incidence of stomach cancer in selected countries: age-standardised rate per 100,000 men (reproduced from http:// globocan.iarc. $\left.f r /{ }^{(2)}\right)$

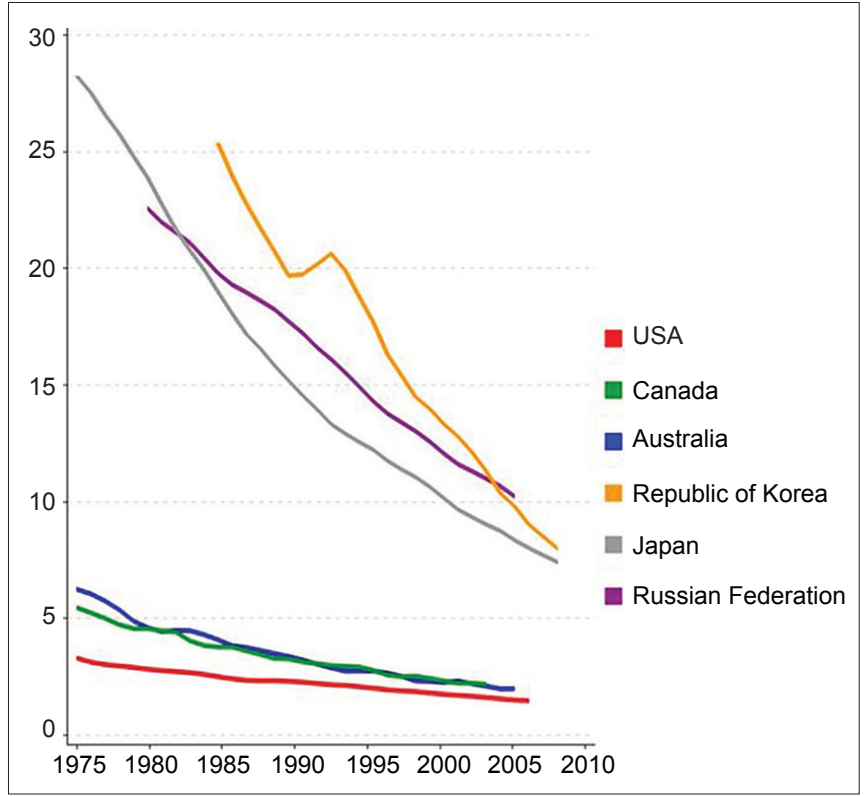

Fig. 3 Graph shows the trends in mortality from stomach cancer in selected countries: age-standardised rate per 100,000 men (reproduced from http:// globocan.iarc. $f r /^{(2)}$ )

cancer arises as a consequence of a complex interaction between host factors, environmental factors and $H$. pylori infection. These specific risk factors will be examined in detail in later paragraphs. The reduction in gastric cancer mortality mainly reflects the decrease in incidence rates. Early cancer detection is important because countries that perform gastric cancer surveillance, such as Japan and Korea, have lower mortality rates, reflecting higher detection rates of early gastric cancer as well as earlier-stage cancers. ${ }^{(2)}$ Surgical and oncologic advances are also important contributory factors of lower gastric cancer mortality rates.

There have been reports indicating that the incidence of gastric cardia cancers is on the rise. ${ }^{(18,19)}$ This is in contrast to the 
trend observed for overall gastric cancer incidence. The rising trend of gastric cardia cancers has been reported in populations from countries with high overall rates of gastric cancer (such as Japan and China), as well as in populations where whites have low overall rates of gastric cancer (such as Denmark and the USA). As a proportion of the total gastric cancer incidence, the contribution from gastric cardia cancers is much higher in low-risk populations. ${ }^{(20)}$ A major risk factor for cardia cancer is obesity, and the increased prevalence rate of obesity, especially in developed countries, could potentially be a contributing factor for this rise in incidence rate. The relationship between obesity and cancer will be further elaborated in this article.

In terms of histology, Lauren's classification subdivided gastric cancer into diffuse (undifferentiated) or intestinal. ${ }^{(21)}$ Distinct clinical and pathological differences have been noted between the two types of gastric cancer. The diffuse type occurs in all age groups with equal gender distribution, unlike the intestinal type, which predominates in males and older persons. The diffuse type involves the corpus or entire stomach, whereas the intestinal type occurs predominantly in the antrum and incisura. In addition, the diffuse type has a greater tendency to invade the gastric wall (resulting in linitis plastic) and to metastatise, and it also has a more rapid disease progression and poorer prognosis. Although both subtypes are related to $H$. pylori infection, the intestinal type undergoes a cascade from atrophic gastritis/ intestinal metaplasia to dysplasia then adenocarcinoma, while the diffuse type dies without following such a cascade. ${ }^{(22)}$ There is also a rare genetically related type called hereditary diffuse gastric cancer (HDGC), which is unrelated to $H$. pylori infection. Intestinal type adenocarcinoma is the main histological subtype of HDGC. It has been noted that, consistent with the overall decline in gastric cancer incidence in recent decades, there has been a global decline in the incidence of intestinal type gastric cancer. However, the decline in the diffuse type has been more gradual. In some series, although intestinal type gastric cancer remained the predominant subtype, the relative proportion of diffuse type cancer became higher over the course of time, ${ }^{(23-27)}$ but this is not noted in all studies. ${ }^{(28,29)}$ The reasons for this relative increase in the proportion of diffuse type cancer are speculative. Given the differences in the carcinogenesis process, it had been postulated that the change may be the result of a decreased rate of severe chronic gastritis, ${ }^{(28)}$ or due to changes in environmental carcinogenic factors $^{(29)}$ in some populations.

\section{H. PYLORI AND GASTRIC CANCER}

Globally, H. pylori infection affects $50 \%$ of the population. ${ }^{(3)}$ It is the most important causal factor for noncardia gastric adenocarcinoma. An analysis of 12 prospective case-control studies by the Helicobacter and Cancer Collaborative Group $^{(30)}$ showed that for non-cardia cancer, the pooled odds ratio (OR) was 2.97 (95\% Cl 2.34-3.77) for H. pylori infection. Conversely, for cardia cancers, no statistically significant association was demonstrated. When the pooled analysis was restricted to cases occurring at least ten years after the diagnosis of H. pylori, the OR for non-cardia cancer increased to 5.93 (95\% Cl
3.41-10.3), and this OR has been regarded as the best estimate of the relative risk (RR) of non-cardia cancer associated with $H$. pylori infection. Based on the average $\mathrm{H}$. pylori prevalence of $35 \%$ and $85 \%$ in developed and developing countries, respectively, it was estimated that about $65 \%-80 \%$ of non-cardia gastric cancers were attributable to $H$. pylori infection and potentially preventable. ${ }^{(30)}$

Regions with high gastric cancer incidence rate tend to have high seroprevalence rates for $H$. pylori infection. However, there are geographical regions, such as Africa and South Asia (e.g. India), where the seroprevalence rates for $H$. pylori infection are high but the gastric cancer incidence rates are low. This phenomenon has been dubbed the Asian or African enigma, ${ }^{(3,31)}$ and is probably a reflection of the complex host, bacterial and environmental factors at play in gastric carcinogenesis. It has also been suggested that disease manifestation reflects the predominant pattern of gastritis within a geographical region, and that the main factor is the interaction of host and environmental factors such as diet, and not the bacterial strain. ${ }^{(3)}$

The Asian-Pacific Consensus Group recommended the screening and treatment of $H$. pylori as an evidenced-based and reasonable strategy for primary prevention of non-cardia gastric cancer in selected communities where the burden of gastric cancer is high. ${ }^{(32)} \mathrm{H}$. pylori eradication has been shown to impact the precursors of gastric cancer. Mera et al ${ }^{(33)}$ assessed the effect of $H$. pylori eradication therapy on intestinal metaplasia, multifocal atrophy and dysplasia in Colombia, a region with high gastric cancer risk. A histopathology score was utilised to document changes in gastric lesions. Multivariate analysis showed a significant regression in histopathology score as a function of the square of $H$. pylori negative time. Subjects who were $H$. pylori negative had $14.8 \%$ more regression and $13.7 \%$ less progression than patients who were positive at 12 years. ${ }^{(33)}$ A prospective, placebo-controlled, randomised study by Leung et al found that subjects with persistent $H$. pylori infection had a significantly higher risk of progression to intestinal metaplasia than those with successful H. pylori eradication (OR 2.13, 95\% Cl 1.41-3.24). ${ }^{(34)}$ A recent Taiwanese study evaluated the effectiveness of mass eradication of $H$. pylori infection in reducing premalignant gastric lesions. The study reported that mass eradication of H.pylori was effective in significantly reducing the incidence of gastric atrophy resulting from chemoprevention $(77.2 \%$; 95\% $\mathrm{Cl} 72.3 \%-81.2 \%)$, although the reduction in incidence of intestinal metaplasia was not significant. ${ }^{(35)}$ Similarly, two other meta-analyses, ${ }^{(36,37)}$ which addressed the controversial issue regarding the impact of $H$. pylori eradication on gastric atrophy and intestinal metaplasia, showed significant improvement in gastric atrophy but not gastric intestinal metaplasia. Thus, gastric intestinal metaplasia is generally regarded as the point of irreversible histological change. ${ }^{(36,37)}$ Uemura et al, ${ }^{(38)}$ who were the first to provide evidence that $H$. pylori eradication has a direct impact on gastric cancer occurrence, conducted a non-randomised $H$. pylorieradication trial in patients with early gastric cancer treated by endoscopic resection. After a three-year follow-up period, $9 \%$ of the untreated patients developed metachronous gastric cancer, while none of the patients who underwent $H$. pylori 
eradication developed the disease. ${ }^{(38)}$ In another prospective observational study, Uemura et al showed that gastric cancer developed in persons infected with H. pylori, but not in uninfected persons. ${ }^{(39)}$ The pooled data from five randomised placebocontrolled H. pylorieradication trials in $\mathrm{Asia}^{(40-44)}$ showed that with H. pylori eradication, the pooled RR of developing gastric cancer was 0.56 (95\% Cl 0.40-0.8). ${ }^{(32)}$ However, it must be recognised that, prior to eradication, irreversible precancerous lesions may have already arisen, and in such cases, apart from $H$. pylori eradication, a strategy of continued surveillance is required.

\section{DIET AND GASTRIC CANCER}

Salt and food preserved with salt are a risk factor for gastric cancer. Since 1959, salt intake has been reported as a possible risk factor for gastric cancer. ${ }^{(45)}$ The possible mechanisms by which salt is involved in gastric cancer include: (a) it potentiates the colonisation and virulence of $H$. pylori; (b) it changes the protective mucous viscosity protecting the stomach, leading to exposure to carcinogens such as $\mathrm{N}$-nitroso compounds; and (c) it causes inflammatory responses of the gastric epithelium, which may increase epithelial cell proliferation as part of the repair process and the probability of endogenous mutations. ${ }^{(46)}$ The INTERSALT Cooperative Research Group ${ }^{(47)}$ conducted an epidemiological study to assess the respective importance of high salt and nitrate intake for gastric cancer mortality at the population level in 24 countries. Although the study found a significant correlation of gastric cancer mortality with sodium and nitrate intake, the correlation with sodium was stronger than nitrate. ${ }^{(47)}$

In a recent meta-analysis, D'Elia et $\mathrm{al}^{(48)}$ examined the role of salt in gastric carcinogenesis by analysing prospective studies. In the pooled analysis, both 'high' and 'moderately high' salt intake versus 'low' salt intake were significantly associated with increased risk of gastric cancer, with RR of 1.68 (95\% Cl $1.17-2.41)$ and 1.41 (1.03-1.93) respectively; the association was found to be stronger in the Japanese population. ${ }^{(48)}$ Additionally, epidemiological and experimental studies $^{(49)}$ have suggested an increased risk of gastric cancer in relation to the consumption of pickled vegetables in East Asia. This issue was addressed in a meta-analysis ${ }^{(49)}$ comparing the gastric cancer risk between people who consumed pickled vegetables/food and non-consumers or those in the lowest reported category of consumption. The study reported the following association: (a) overall: OR 1.52, 95\% Cl 1.37-1.68; (b) case-control studies: OR 1.56, 95\% Cl 1.39-1.75; and (c) cohort studies: OR 1.32, 95\% Cl 1.10-1.59. The following association was found with studies from different geographical regions: (a) Korean: OR 1.89, 95\% Cl 1.29-2.77; (b) Chinese: OR 1.86, 95\% Cl 1.61-2.15; (c) Japanese: OR 1.16, 95\% Cl 1.04-1.29; and (d) other countries: OR 1.14, 95\% Cl $0.96-1.35){ }^{(49)}$

Conversely, several prospective studies have reported significant reductions in gastric cancer risk (both cardia and non-cardia) arising from the consumption of fresh fruits and vegetables, the results of which were summarised in a recent meta-analysis. ${ }^{(50)}$ An inverse association was found between fruit intake and gastric cancer incidence (RR 0.82, 95\% Cl
0.73-0.93), and this was stronger for follow-up periods of more than ten years (RR $0.66,95 \% \mathrm{Cl} 0.52-0.83$ ). For vegetables, the RR were 0.88 (95\% Cl 0.69-1.13) and 0.71 (95\% Cl 0.53-0.94) when considering all incidence studies and studies with a longer follow-up, respectively. Bertuccio et al ${ }^{(51)}$ analysed the impact of dietary pattern, in terms of a prudent/healthy diet rich in fruits and vegetables versus a Western/unhealthy diet rich in starchy foods, meat and fats. The former diet had an OR of $0.75(95 \%$ Cl 0.63-0.90) for gastric cancer for the highest versus the lowest category. The Western/unhealthy dietary pattern had an OR of 1.51 (95\% Cl 1.21-1.89) for gastric cancer. It was concluded that there was a twofold difference in gastric cancer risk between a prudent/healthy diet rich in fruits and vegetables, and a Western/ unhealthy diet rich in starchy foods, meat and fats. ${ }^{(51)}$

Green tea, made from the plant Camellia sinensis, contains the active ingredient polyphenol, which has a subgroup known as catechins. Catechins are powerful antioxidants that may inhibit cell proliferation and prevent cancer. Data regarding the effect of green tea on prevention of gastric cancer is conflicting, with no clear benefit. ${ }^{(52)}$ A recent Japanese study that analysed pooled data from six cohort studies suggested that, in women, a significantly decreased risk of distal gastric cancer was observed in those who consumed more than five cups of green tea per day (hazard ratio $0.70,95 \% \mathrm{Cl} 0.50-0.96)$, but such an association was not seen in men. ${ }^{(53)}$ Therefore, although there are clear epidemiological associations between diet and gastric cancer, the role of dietary interventions remains unproven.

\section{LIFESTYLE AND GASTRIC CANCER}

Two lifestyle factors implicated in gastric carcinogens are smoking and alcohol. The evidence for smoking as a risk factor is unequivocal. Comparing current smokers with non-smokers, the RR was 1.62 in males $(95 \% \mathrm{Cl} 1.50-1.75)$ and 1.20 in females (95\% Cl 1.01-1.43); the RR increased from 1.3 for the lowest consumption to 1.7 for consumption of approximately 30 cigarettes per day. Smoking was significantly associated with both cardia (RR 1.87, 95\% Cl 1.31-2.67) and non-cardia (RR 1.60, 95\% Cl 1.41-1.80) cancers. ${ }^{(54)}$ In contrast, no definite epidemiological association exists between alcohol and gastric cancer. ${ }^{(55)}$

\section{METABOLIC RISK FACTORS FOR GASTRIC CANCER}

The relationship between serum cholesterol levels and gastric cancer is controversial, with conflicting data from case control and cohort studies. Explanations for causality are speculative, especially since both low and high cholesterol levels have been associated with gastric cancer. Asano et $\mathrm{al}^{(56)}$ conducted a prospective population-based study to examine the association between serum cholesterol levels and the incidence of gastric cancer. It was found that the risk of gastric cancer increased with decreasing cholesterol levels, even after adjustment for other confounding factors such as age, gender, H. pylori infection, atrophic gastritis, family history of malignant neoplasm, smoking habits, body mass index (BMI) and dietary factors. This 
association was significant for intestinal type gastric cancers, but not for the diffuse type. Asano et al suggested that since there was experimental evidence that long-term depletion of serum cholesterol-induced nuclear factor kappa B activation, which could promote tumourigenesis, low cholesterol levels could have a causal effect on one of the processes of carcinogenesis. ${ }^{(56)}$ However, no association with low serum cholesterol was noted in other prospective population studies. ${ }^{(57,58)}$

Another large prospective Japanese study, which evaluated the inverse relationship between serum cholesterol and gastric cancer, showed that although serum total cholesterol levels had strong inverse associations with stomach cancer in men, after exclusion for the first three-year incident cases and advanced cases with metastasis, the inverse association diminished for stomach cancers. ${ }^{(59)}$ In the Japanese study, a possible confounding factor could be the impact of existing undiagnosed cancer on cholesterol level at the time of testing. On the other hand, a possible relationship between high cholesterol levels and gastric cancer has also been reported. For instance, a recent questionnaire survey assessing the dietary habits of cancer patients reported that high dietary cholesterol was positively associated with the risk of stomach and other cancers. ${ }^{(60)}$ Also, Jung et al showed in a small case-control study that the risk for dysplasia was increased among persons with hyperglycaemia and a higher level of low-density lipoprotein (LDL) cholesterol, although BMI, triglyceride level and total cholesterol level were not associated with the risk for dysplasia. ${ }^{(61)}$ Postulated mechanisms to explain the relationship between hyperlipidaemia and gastric cancer include the impact of hyperlipidaemia on cellular inflammation, impact on levels of proinflammatory cytokines, and the role of iron-induced oxidation of serum lipids. ${ }^{(60,61)}$

Another study by Ikeda et $\mathrm{al}^{(62)}$ evaluated the impact of haemoglobin A1c (HbA1c) levels on the occurrence of gastric cancer, and their interaction with $H$. pylori infection. It was found that the age- and gender-adjusted incidence of gastric cancer was significantly increased when $\mathrm{HbA1c}$ was higher than 6, even after adjusting for confounding factors, including $H$. pylori seropositivity. In addition, this risk was further increased in the presence of $\boldsymbol{H}$. pylori infection. ${ }^{(62)}$ The precise pathogenetic role of hyperglycaemia in gastric carcinogenesis is obscure, but postulated mechanisms include increased production of reactive oxygen species and greater oxidative damage to DNA, as well as the impact of hyperinsulinaemia on cellular proliferation. ${ }^{(62)}$ A recent Korean case-control study ${ }^{(63)}$ examined the relationship between metabolic syndrome and gastric cancer. Multivariate analysis revealed that fasting glucose, total cholesterol, LDLcholesterol and metabolic syndrome had a statistically significant relationship with gastric dysplasia. The risk of gastric dysplasia was associated with the following: (a) modest glucose levels (glucose 100-125 mg/dL; age- and gender-adjusted RR 2.261, 95\% Cl 1.147-4.457); (b) higher total cholesterol levels (total cholesterol $\geq 240 \mathrm{mg} / \mathrm{dL}$; RR 6.299, 95\% Cl 1.277-31.076); (c) modest LDL-cholesterol levels (LDL 130-159 mg/dL; RR $0.250,95 \% \mathrm{Cl} 0.069-0.903)$; and (d) the presence of metabolic syndrome (RR 2.177, 95\% Cl 1.082-4.379). ${ }^{(63)}$ Given the association between cholesterol/metabolic syndrome with gastric cancer, a pertinent issue would be the impact of statins on gastric cancer risk. A meta-analysis examined 26 randomised control trials involving 290 gastric cancers and eight observational studies totalling 7,321 gastric cancers. Statins use was shown to be significantly associated with a $27 \%$ reduction in gastric cancer risk (RR 0.73, 95\% Cl 0.58-0.93). ${ }^{(64)}$

To summarise, the relationship between gastric cancer and serum cholesterol or serum glucose levels remains controversial, with no proof of causality. As in the case of dietary intervention, it is therefore premature to recommend the use of statins or strategies targeting these metabolic factors for gastric cancer prevention.

\section{OBESITY AND GASTRIC CANCER}

Obesity is a major risk factor for gastric cardia adenocarcinoma. A meta-analysis showed that obesity resulted in an elevated risk for cardia gastric cancer, with the following summary: (a) overweight subjects (BMI 25-30 kg/m²): estimated RR 1.4, 95\% Cl 1.16-1.68; and (b) obese subjects $\left(\mathrm{BMI}>30 \mathrm{~kg} / \mathrm{m}^{2}\right)$ : estimated RR 2.06, 95\% Cl 1.63-2.61. ${ }^{(65)}$ However, no increased risk has been found for non-cardia gastric cancer. ${ }^{(66,67)}$ The exact pathological mechanism is yet unclear, and postulated mechanisms include increased risk of oesophageal reflux, insulin resistance, levels of adiponectin and leptin, insulin-like growth factors, sex steroids and glucocorticoids, obesity-related inflammatory markers, and oxidative stresses. ${ }^{(65)}$

\section{GASTRIC CANCER AND FAMILIAL ASSOCIATIONS}

Gastric cancer and familial associations may be secondary to either shared environmental exposure or true genetic susceptibility. El-Omar et al $^{(68)}$ examined the prevalence of atrophy and hypochlorhydria, and their associations with $H$. pylori infection in first-degree relatives of patients with gastric cancer. Among these relatives, the prevalence of atrophy and hypochlorhydria was increased only in those with evidence of H. pylori infection. Eradication of H. pylori infection produced resolution of the gastric inflammation in each subject, and resolution of hypochlorhydria and atrophy in 50\% of the subjects. ${ }^{(68)}$ Brenner et al ${ }^{(69)}$ carried out a population-based, statewide, case-control study in Germany to assess the individual and joint contributions of family history and $H$. pylori infection to the risk of gastric carcinoma. It was found that, although H. pylori infection and family history were positively related, both were independently and strongly associated with a risk for gastric cancer. Compared with uninfected subjects without a family history, subjects with both a positive family history and infection with cagA-positive H. pylori strains had an OR of $8.2(95 \% \mathrm{Cl}$ $2.2-30.4)$ for gastric cancer overall and an OR of $16(95 \% \mathrm{Cl}$ 3.9-66.4) for non-cardia gastric cancer. ${ }^{(69)}$

HDGC is a rare cancer, representing approximately $2 \%$ of all gastric cancers. It is caused by specifically associated germline mutations to the E-cadherin ( $C D H 1)$ gene. HDGC is characterised by autosomal dominance and high penetrance, as well as a high 
cumulative risk for advanced gastric cancer. In this rare disorder, $\mathrm{CDH} 1$ mutation testing in families with a history of gastric cancer and prophylactic gastrectomy in mutation-positive patients are recommended for management. ${ }^{(70)}$

\section{HOST GENETIC POLYMORPHISMS AND GASTRIC CANCER RISK}

Host genetic polymorphisms may have an impact on host responses to gastric inflammation and acid secretion, thereby interacting with H. pylori infection in gastric carcinogenesis. A series of single nucleotide polymorphisms (SNPs) of susceptibility genes have been implicated. El-Omar et al ${ }^{(71)}$ were the first to report that interleukin-1 (i.e. $/ L 1 B$ ) gene cluster polymorphisms, suspected of enhancing production of $I L 1 B$, are associated with an increased risk of both hypochlorhydria-induced $H$. pylori and gastric cancer. $I L 1 B$ is an important pro-inflammatory cytokine and a powerful inhibitor of gastric acid secretion. Host genetic factors that affect IL 1B may determine why some individuals infected with $H$. pylori develop gastric cancer, while others do not. ${ }^{(71)}$ The inflammatory response-related genes that have been most frequently studied are interleukin genes $I L 1 B, I L I R N, I L 8$ and $I L 10$, and tumour necrosis factor-alpha (TNFA), coding for the proteins IL- $1 \mathrm{~b}$, IL-1 ra, IL-8, IL-10, and TNF-a, respectively. However, the results of these studies are not consistent across Asian and non-Asian populations. A recent meta-analysis ${ }^{(72)}$ examined the relationship between these genetic polymorphisms and gastric cancer risk. Overall, the study showed statistically significant associations in IL1RN2 and IL10-592A carriers. In studies from Asian populations, an increased risk of gastric cancer among IL10-1082G carriers and a decreased risk among IL1B-31C carriers were observed. Studies from non-Asian populations showed an increased risk among IL1RN2 carriers and a decreased risk among IL10-1082G carriers. Furthermore, studies from non-Asian populations also observed increased risks among carriers of IL1B-511T, IL1B-31C and IL1RN2 for cardia gastric cancer, but this was not statistically significant in Asian populations. In Asian populations, IL1B-31C carriers were found to have a decreased risk and IL1RN2 carriers an increased risk for non-cardia gastric cancer. An increased risk was shown in carriers of the IL1B-511T, IL1RN2 and TNFA-308A alleles among non-Asian populations. In studies reporting results for intestinal type gastric cancer, Asian populations showed a statistically significant decreased risk among IL1B-511T and IL1B31C carriers, and an increased risk among non-Asian IL1RN2 carriers. For studies from non-Asian populations, increased risks for IL1RN2 and TNFA-308A carriers and a decreased risk of diffuse type cancer among IL10-1082G carriers were found. ${ }^{(72)}$

\section{MOLECULAR SUBTYPES OF GASTRIC CANCER}

The Lauren classification of gastric cancer is widely used, as it distinguishes two main subtypes that are biologically different, with distinct clinical and epidemiological profiles. ${ }^{(21)}$ However, in clinical practice, this classification is not useful for predicting treatment response or survival. A robust molecular classification system, based on genomic/molecular analysis, could potentially provide insights into oncogenic mechanisms and identify unique molecular drivers that could be correlated with survival and treatment response. Building upon prior efforts in microarraygene expression profiling to discover molecular subtypes of gastric cancer that were limited by small sample sizes, Lei et $\mathrm{al}^{(73)}$ compared gene expression patterns in 248 Singapore cases of gastric cancer using hierarchical clustering with iterative feature selection. The study identified three molecular signatures, which were further validated in 70 cases of gastric cancer from Australia. The first subtype had high activity of the epithelialmesenchymal transition pathway, and was hence termed mesenchymal. The second subtype was termed proliferative because it was characterised by gene sets associated with the cell cycle; it was also associated with high activities for several oncogenic pathways such as E2F, MYC and RAS. The third subtype was termed metabolic, as it was characterised by gene sets from several KEGG metabolism pathways. Interestingly, the mesenchymal and proliferative subtypes largely coincided with Lauren's diffuse and intestinal subtypes (70\% and $75 \%$, respectively), whereas the metabolic subtype was nearly evenly split between diffuse and intestinal subtypes. However, the metabolic subtype had features of spasmolytic polypeptideexpressing metaplasia. In terms of therapeutic implications, the key finding of Lei et al's study was that the metabolic subtype had in vitro sensitivity to 5-fluorouracil, which also appeared to be true in vivo, based on correlation with actual clinical data in Singapore. The mesenchymal subtype was found to be more sensitive to drugs that target the phosphatidylinositol-3 kinaseAKT-mTOR pathway in vitro, although in vivo patient data were not available. ${ }^{(73)}$ This study represents hope for molecularly tailored personalised therapies for gastric adenocarcinoma.

\section{CONCLUSION}

Although the overall incidence rate of gastric cancer continues to fall, it still remains clinically relevant because gastric cancer is still the second leading cause of cancer-related mortality and the fourth most common cancer globally. Gastric carcinogenesis arises as a consequence of a complex interaction between host and environmental factors. $H$. pylori infection is the major risk factor associated with non-cardia gastric cancer, and data has emerged with regard to the role of $H$. pylori eradication for primary prevention of gastric cancer. Smoking has also been implicated as a risk factor for non-cardia cancer. Furthermore, host genetic polymorphisms have an impact on host responses to gastric inflammation and acid secretion, thereby interacting with $H$. pylori infection and other environmental factors in gastric carcinogenesis. In contrast to non-cardia cancer, H. pylori infection does not play an important role in cardia cancer, with obesity and smoking identified as the main risk factors. Although dietary, lifestyle and metabolic risk factors have been identified, and addressing these lifestyle and metabolic risk factors may contribute to health, the actual impact in terms of cancer prevention is unclear. In the overall scheme of management, H. pylori infection represents a target for primary prevention, but once irreversible histological changes have occurred, endoscopic 
surveillance would be required, all in the hope of detection of early gastric cancer that is amenable to curative endoscopic resection, or earlier stage disease that is surgically curable. For more advanced cancer requiring chemotherapy, a robust molecular classification system represents hope for molecularly tailored personalised therapies, which may improve prognosis.

\section{REFERENCES}

1. Ferlay J, Shin HR, Bray F, et al. Estimates of worldwide burden of cancer in 2008: GLOBOSCAN 2008. Int J Cancer 2010; 127:2893-917.

2. Ferlay J, Shin HR, Bray F, et al. GLOBOCAN 2008 v2.0, Cancer Incidence and Mortality Worldwide: IARC CancerBase No. 10 [Online]. Available: http://globocan.iarc.fr. Accessed June 3, 2014.

3. Fock KM, Ang TL. Epidemiology of Helicobacter pylori infection and gastric cancer in Asia. J Gastroenterol Hepatol 2010; 25:479-86.

4. Wong BC, Lm SK, Ching CK, et al. Differential Helicobacter pylor infection rates in two contrasting gastric cancer risk regions of South China. China Gastric Cancer Study Group. J Gastroenterol Hepatol 1999; 14:120-5.

5. Zhang DH, Zhou LY, Lin SR, et al. Recent changes in the prevalence of Helicobacter pylori infection among children and adults in high- or lowincidence regions of gastric cancer in China. Chin Med J (Engl) 2009; 122:1759-63.

6. Aragonés N, Pérez-Gómez B, Pollán $M$, et al. The striking geographical pattern of gastric cancer mortality in Spain: environmental hypotheses revisited. BMC Cancer 2009; 9:316.

7. Mezzanotte G, Cislaghi C, Decarli A, La Vecchia C. Cancer mortality in broad Italian geographical areas, 1975-1977. Tumori 1986; 72:145-52.

8. Wong BC, Ching CK, Lam SK, et al. Differential north to south gastric cancer-duodenal ulcer gradient in China. China Ulcer Study Group. J Gastroenterol Hepatol 1998; 13:1050-57.

9. Arnold M, Razum O, Coebergh JW. Cancer risk diversity in non-western migrants to Europe: An overview of the literature. Eur J Cancer 2010; 46:2647-59.

10. Ronellenfitsch $U$, Kyobutungi C, Ott JJ, et al. Stomach cancer mortality in two large cohorts of migrants from the Former Soviet Union to Israel and Germany: are there implications for prevention? Eur J Gastroenterol Hepatol 2009; 21:409-16.

11. Nguyen EV. Cancer in Asian American males: epidemiology, causes, prevention, and early detection. Asian Am Pac IsI J Health 2003; 10:86-99.

12. Maskarinec G, Noh JJ. The effect of migration on cancer incidence among Japanese in Hawaii. Ethn Dis 2004; 14:431-9.

13. Bertuccio $P$, Chatenoud $L$, Levi $F$, et al. Recent patterns in gastric cancer: a global overview. Int J Cancer 2009; 125:666-73.

14. Matsuda A, Machii R. Trends in stomach cancer mortality rates in Japan, USA, UK, France and Korea based on the WHO mortality database. Jpn J Clin Oncol 2012; 42:154

15. Tanaka M, Ma E, Tanaka $\mathrm{H}$, et al. Trends of stomach cancer mortality in Eastern Asia in 1950-2004: comparative study of Japan, Hong Kong and Singapore using age, period and cohort analysis. Int J Cancer 2012 130:930-6.

16. Anderson WF, Camargo MC, Fraumeni JF Jr, et al. Age-specific trends in incidence of noncardia gastric cancer in US adults. JAMA 2010; 303:1723-8.

17. Bray F, Jemal A, Grey N, Ferlay J, Forman D. Global cancer transitions according to the Human Development Index (2008-2030): a populationbased study. Lancet Oncol 2012; 13:790-801.

18. Devesa SS, Blot WJ, Fraumeni JF Jr. Changing patterns in the incidence of esophageal and gastric carcinoma in the United States. Cancer 1998; 83:2049-53.

19. Botterweck AA, Schouten LJ, Volovics A, Dorant E, van Den Brandt PA. Trends in incidence of adenocarcinoma of the oesophagus and gastric cardia in ten European countries. Int J Epidemiol 2000; 29:645-54.

20. de Martel C, Forman D, Plummer M. Gastric cancer: epidemiology and risk factors. Gastroenterol Clin North Am 2013; 42:219-40.

21. Lauren $P$. The two histological main types of gastric carcinoma: diffuse and so-called intestinal-type carcinoma. An attempt at a histo-clinical classification. Acta Pathol Microbiol Scand 1965; 64:31-49.

22. Vauhkonen $M$, Vauhkonen $H$, Sipponen P. Pathology and molecular biology of gastric cancer. Best Pract Res Clin Gastroenterol 2006; 20:651-74.
23. Laurén PA, Nevalainen TJ. Epidemiology of intestinal and diffuse types of gastric carcinoma. A time-trend study in Finland with comparison between studies from high- and low-risk areas. Cancer 1993; 71:2926-33.

24. Ikeda Y, Mori M, Kamakura T, et al. Improvements in diagnosis have changed the incidence of histological types in advanced gastric cancer. $\mathrm{Br}$ J Cancer 1995; 72:424-6.

25. Yu J, Zhao Q. The demographic characteristics of histological types of gastric cancer with gender, age, and tumor location. J Gastrointest Cancer 2009; 40:98-100.

26. Popiela T, Kulig J, Kołodziejczyk P, Sierzega M. Changing patterns of gastric carcinoma over the past two decades in a single institution: clinicopathological findings in 1557 patients. Scand J Gastroenterol 2002; 37:561-7.

27. Henson DE, Dittus C, Younes M, Nguyen H, Albores-Saavedra J. Differential trends in the intestinal and diffuse types of gastric carcinoma in the United States, 1973-2000: increase in the signet ring cell type. Arch Pathol Lab Med 2004; 128:765-70.

28. Lundegårdh G, Lindgren A, Rohul A, et al. Intestinal and diffuse types of gastric cancer: secular trends in Sweden since 1951. Br J Cancer 1991; 64:1182-6.

29. Kaneko S, Yoshimura T. Time trend analysis of gastric cancer incidence in Japan by histological types, 1975-1989. Br J Cancer 2001; 84:400-5.

30. Helicobacter and Cancer Collaborative Group. Gastric cancer and Helicobacter pylori: a combined analysis of 12 case control studies nested within prospective cohorts. Gut 2001; 49:347-53.

31. Graham DY, Lu H, Yamaoka Y. African, Asian or Indian enigma, the East Asian Helicobacter pylori: facts or medical myths. J Dig Dis 2009; 10:77-84.

32. Fock KM, Talley N, Moayyedi $P$, et al. Asia-Pacific consensus guidelines on gastric cancer prevention. J Gastroenterol Hepatol 2008; 23:351-65.

33. Mera R, Fontham ET, Bravo LE, et al. Long term follow up of patients treated for Helicobacter pylori infection. Gut 2005; 54:1536-40.

34. Leung WK, Lin SR, Ching JY, et al. Factors predicting progression of gastric intestinal metaplasia: results of a randomized trial on Helicobacter pylori eradication. Gut 2004; 53: 1244-9.

35. Lee YC, Chen TH, Chiu HM, et al. The benefit of mass eradication of Helicobacter pylori infection: a community-based study of gastric cancer prevention. Gut 2013; 62:676-82.

36. Rokkas T, Pistiolas D, Sechopoulos P, Robotis I, Margantinis G. The long-term impact of Helicobacter pylori eradication on gastric histology: a systematic review and meta-analysis. Helicobacter 2007; 12 Suppl 2:32-8.

37. Wang J, Xu L, Shi R, et al. Gastric atrophy and intestinal metaplasia before and after Helicobacter pylori eradication: a meta-analysis. Digestion 2011; 83:253-60.

38. Uemura N, Mukai T, Okamoto S, et al. Effect of Helicobacter pylori eradication on subsequent development of cancer after endoscopic resection of early gastric cancer. Cancer Epidemiol Biomarkers Prev 1997; 6:639-42.

39. Uemura N, Okamoto S, Yamamoto S, et al. Helicobacter pylori infection and the development of gastric cancer. N Engl J Med 2001; 345:784-9.

40. You WC, Brown LM, Zhang L, et al. Randomized double-blind factorial trial of three treatments to reduce the prevalence of precancerous gastric lesions. J Natl Cancer Inst 2006; 98:974-83.

41. Zhou LY, Lin SR, Ding SG, et al. The changing trends of the incidence of gastric cancer after Helicobacter pylori eradication in Shandong area. Chin J Dig Dis 2005; 6:114-5.

42. Wong BC, Lam SK, Wong WM, et al. Helicobacter pylori eradication to prevent gastric cancer in a high-risk region of China: a randomized controlled trial. JAMA 2004; 291:187-94.

43. Saito D, Boku N, Fujioka T, et al. Impact of H. pylori eradication on gastric cancer prevention: endoscopic results of the Japanese Intervention Trial (JITHP-Study). A Randomized Multi-Center Trial. Gastroenterology 2005; 128 Suppl 2:A4.

44. Kato M, Asaka M. Eradication of Helicobacter pylori for the incidence of metachronous gastric cancer after endoscopic resection. J Gastroenterol Hepatol 2007; 22:A64.

45. Sato T, Fukuyama T, Suzuki T, et al. Studies of causation of gastric cancer 2. The relation between gastric cancer mortality rate and salted food intake in several places in Japan. Bull Inst Public Health 1959; 8:187-98.

46. Wang XQ, Terry PD, Yan H. Review of salt consumption and stomach cancer risk: epidemiological and biological evidence. World J Gastroenterol 2009; 15:2204-13.

47. Joossens JV, Hill MJ, Elliott P, et al. Dietary salt, nitrate and stomach cancer mortality in 24 countries. European Cancer Prevention (ECP) and the INTERSALT Cooperative Research Group. Int J Epidemiol 1996; 25:494-504. 
48. D’Elia L, Rossi G, Ippolito R, Cappuccio FP, Strazzullo P. Habitual salt intake and risk of gastric cancer: a meta-analysis of prospective studies. Clin Nutr 2012; 31:489-98.

49. Ren JS, Kamangar F, Forman D, Islami F. Pickled food and risk of gastric cancer--a systematic review and meta-analysis of English and Chinese literature. Cancer Epidemiol Biomarkers Prev 2012; 21:905-15.

50. Lunet N, Lacerda-Vieira A, Barros H. Fruit and vegetables consumption and gastric cancer: a systematic review and meta-analysis of cohort studies Nutr Cancer 2005; 53:1-10.

51. Bertuccio $P$, Rosato $V$, Andreano A, et al. Dietary patterns and gastric cance risk: a systematic review and meta-analysis. Ann Oncol 2013; 24:1450-8.

52. Boehm K, Borrelli F, Ernst E, et al. Green tea (Camellia sinensis) for the prevention of cancer. Cochrane Database Syst Rev 2009; 3:CD005004.

53. Sasazuki S, Tamakoshi A, Matsuo K, et al. Green tea consumption and gastric cancer risk: an evaluation based on a systematic review of epidemiologic evidence among the Japanese population. Jpn J Clin Onco 2012; 42:335-46

54. Ladeiras-Lopes R, Pereira AK, Nogueira A, et al. Smoking and gastric cancer: systematic review and meta-analysis of cohort studies. Cance Causes Control 2008; 19:689-701.

55. Shimazu T, Tsuji I, Inoue M, et al. Alcohol drinking and gastric cancer risk: an evaluation based on a systematic review of epidemiologic evidence among the Japanese population. Jpn J Clin Oncol 2008; 38:8-25.

56. Asano K, Kubo M, Yonemoto K, et al. Impact of serum total cholesterol on the incidence of gastric cancer in a population-based prospective study: the Hisayama study. Int J Cancer 2008; 122:909-14.

57. Stemmermann GN, Chyou PH, Kagan A, Nomura AM, Yano K. Serum cholesterol and mortality among Japanese-American men. The Honolulu (Hawaii) Heart Program. Arch Intern Med 1991; 151:969-72.

58. Eichholzer M, Stähelin HB, Gutzwiller F, Lüdin E, Bernasconi F. Association of low plasma cholesterol with mortality for cancer at various sites in men: 17-y follow-up of the prospective Basel study. Am J Clin Nutr 2000; 71:569-74

59. Iso H, Ikeda A, Inoue M, Sato S, Tsugane S. Serum cholesterol levels in relation to the incidence of cancer: the JPHC study cohorts. Int J Cance 2009; 125:2679-86.

60. Hu J, La Vecchia C, de Groh M, et al. Dietary cholesterol intake and cancer. Ann Oncol 2012; 23:491-500.
61. Jung MK, Jeon SW, Cho CM, et al. Hyperglycaemia, hypercholesterolemia and the risk for developing gastric dysplasia. Dig Liver Dis 2008; 40:361-5.

62. Ikeda F, Doi Y, Yonemoto K, et al. Hyperglycemia increases risk of gastric cancer posed by Helicobacter pylori infection: a population-based cohort study. Gastroenterology 2009; 136:1234-41.

63. Kim HY. Metabolic syndrome is associated with gastric dysplasia. Eur J Gastroenterol Hepatol 2011; 23:871-5

64. Wu XD, Zeng K, Xue FQ, Chen JH, Chen YQ. Statins are associated with reduced risk of gastric cancer: a meta-analysis. Eur J Clin Pharmacol 2013; 69:1855-60

65. Yang $\mathrm{P}$, Zhou $\mathrm{Y}$, Chen B, et al. Overweight, obesity and gastric cancer risk: results from a meta-analysis of cohort studies. Eur J Cancer 2009; 45:2867-73.

66. Sjodahl K, Jia C, Vatten L, et al. Body mass and physical activity and risk of gastric cancer in a population-based cohort study in Norway. Cancer Epidemiol Biomarkers Prev 2008; 17:135-40.

67. Kuriyama S, Tsubono Y, Hozawa A, et al. Obesity and risk of cancer in Japan. Int J Cancer 2005; 113:148-57.

68. El-Omar EM, Oien K, Murray LS, et al. Increased prevalence of precancerous changes in relatives of gastric cancer patients: critical role of H. pylori. Gastroenterology 2000; 118:22-30.

69. Brenner H, Arndt V, Stürmer T, et al. Individual and joint contribution of family history and Helicobacter pylori infection to the risk of gastric carcinoma. Cancer 2000; 88:274-9.

70. Seevaratnam R, Coburn N, Cardoso R, et al. A systematic review of the indications for genetic testing and prophylactic gastrectomy among patients with hereditary diffuse gastric cancer. Gastric Cancer 2012; 15 Suppl 1:S153-63.

71. El-Omar EM, Carrington M, Chow WH, et al. Interleukin-1 polymorphisms associated with increased risk of gastric cancer. Nature 2000; 404:398-402.

72. Persson C, Canedo P, Machado JC, El-Omar EM, Forman D. Polymorphisms in inflammatory response genes and their association with gastric cancer: A HuGE systematic review and meta-analyses. Am J Epidemiol 2011; 173:259-70

73. Lei Z, Tan IB, Das K, et al. Identification of molecular subtypes of gastric cancer with different responses to PI3-kinase inhibitors and 5-fluorouracil. Gastroenterology 2013; 145:554-65. 